Meta

Journal des traducteurs

Translators' Journal

\title{
Intertextualité et traduction
}

\section{Geneviève Roux-Faucard}

Volume 51, numéro 1, mars 2006

URI : https://id.erudit.org/iderudit/012996ar

DOI : https://doi.org/10.7202/012996ar

Aller au sommaire du numéro

\section{Éditeur(s)}

Les Presses de l'Université de Montréal

ISSN

0026-0452 (imprimé)

1492-1421 (numérique)

Découvrir la revue

Citer cet article

Roux-Faucard, G. (2006). Intertextualité et traduction. Meta, 51(1), 98-118.

https://doi.org/10.7202/012996ar

\section{Résumé de l'article}

Le sens d'un texte ne se constitue pas uniquement dans sa relation à l'auteur et au lecteur (lecteur implicite, lecteur réel), mais aussi dans sa relation à d'autres textes. Rencontrées dans un texte à traduire, les traces intertextuelles (citations, allusions, références) posent un problème spécifique. Cette difficulté est particulièrement sensible lorsque le texte cité par l'original n'est pas familier à la culture d'accueil. Le traducteur peut se voir amené à intervenir par des pratiques explicitatives, risquant alors de modifier l'effet produit ou visé. Une autre solution consiste à privilégier la fonction du lien intertextuel ou à effectuer une adaptation.

Par le jeu de l'intertextualité, chaque texte prend sa place à l'intérieur d'un vaste réseau. La seconde partie de l'article montre que le texte traduit y a, lui aussi, sa place, qui n'est pas la même que celle de son texte directeur. Le traducteur doit accepter cette donnée qui, loin de limiter la valeur d'une traduction, fait d'elle un texte vivant, autonome, et constitue peut-être la condition d'existence d'une " grande " traduction.

Cette étude s’appuie sur différents exemples empruntés à la littérature allemande (Kafka, Fontane, Zweig), avec plusieurs traductions françaises et anglaises.
Ce document est protégé par la loi sur le droit d'auteur. L'utilisation des services d'Érudit (y compris la reproduction) est assujettie à sa politique d'utilisation que vous pouvez consulter en ligne.

https://apropos.erudit.org/fr/usagers/politique-dutilisation/ 


\title{
Intertextualité et traduction
}

\author{
GENEVIÈVE ROUX-FAUCARD \\ Université de Paris III (ESIT), Paris, France \\ genevieve.roux@free.fr
}

\section{RÉSUMÉ}

Le sens d'un texte ne se constitue pas uniquement dans sa relation à l'auteur et au lecteur (lecteur implicite, lecteur réel), mais aussi dans sa relation à d'autres textes. Rencontrées dans un texte à traduire, les traces intertextuelles (citations, allusions, références) posent un problème spécifique. Cette difficulté est particulièrement sensible lorsque le texte cité par l'original n'est pas familier à la culture d'accueil. Le traducteur peut se voir amené à intervenir par des pratiques explicitatives, risquant alors de modifier l'effet produit ou visé. Une autre solution consiste à privilégier la fonction du lien intertextuel ou à effectuer une adaptation.

Par le jeu de l'intertextualité, chaque texte prend sa place à l'intérieur d'un vaste réseau. La seconde partie de l'article montre que le texte traduit y a, lui aussi, sa place, qui n'est pas la même que celle de son texte directeur. Le traducteur doit accepter cette donnée qui, loin de limiter la valeur d'une traduction, fait d'elle un texte vivant, autonome, et constitue peut-être la condition d'existence d'une «grande» traduction.

Cette étude s'appuie sur différents exemples empruntés à la littérature allemande (Kafka, Fontane, Zweig), avec plusieurs traductions françaises et anglaises.

\section{ABSTRACT}

Intertextuality means that a text is not only related to the author and the reader, but also to other texts. It contains intertextual links, such as quotations, allusions and references, which are especially difficult to translate. By translating them literally, the meaning may be lost. The translator has to help the reader by providing explanations, notes and so on, but this can change the overall effect of the text. The problem is especially acute when the quoted text is not well-known in the target culture. One possible solution is to stress the function of the intertextual links; another is adaptation.

Each text exists within a general network which is like a vast society of texts. In the second part of the article, it is shown that a translated text has its own place on the intertextual web, which can never be the same as that of the original. The translator must accept this reality, which turns a translation into a living work. Intertextuality and translation enjoy a close and very fruitful relationship.

This study is illustrated by several examples taken from German literature (Kafka, Fontane, Zweig) with various translations into French and English.

\section{MOTS-CLÉS/KEYWORDS}

allusion, citation, intertextualité, transfert culturel

Parmi les outils critiques servant à l'analyse textuelle, l'intertextualité, définie par D. Maingueneau ${ }^{1}$ comme «ensemble de relations avec d'autres textes se manifestant à l'intérieur d'un texte», est une notion relativement récente, mais déjà couramment utilisée. Dès cette première définition, on conçoit en effet qu'elle soit indispensable à ceux qui ont mission de décrire et d'évaluer le texte: critique littéraire, historien de la littérature, théoricien de la création littéraire, analyste du discours. Mais qu'en est- 
il pour le lecteur et pour cette sorte particulière de lecteur qu'est le traducteur? Avant de répondre, un bref rappel historique nous permettra de préciser les contours du sujet.

Le mot et la notion d'intertextualité apparaissent au sein du groupe d'avant-garde Tel Quel. Philippe Sollers ${ }^{2}$ : "Tout texte se situe à la jonction de plusieurs textes dont il est à la fois la relecture, l'accentuation, la condensation, le déplacement et la profondeur.» Julia Kristeva ${ }^{3}$ : "Tout texte se construit comme mosaïque de citations, tout texte est absorption et transformation d'un autre texte.» De l'aveu même de ces auteurs, la notion est née de l'idée de "polyphonie», développée par Bakhtine, dont l'œuvre fut écrite dans les années 1920, mais ne fut traduite et interprétée en France que vers la fin des années 1960.

Au cours de la décennie suivante, la notion est rapidement reprise et diffusée. Elle fait l'objet d'un inventaire tous azimuts, où la matière transformée est tantôt discours idéologique (Barthes), tantôt contenu de l'inconscient (Kristeva), tantôt phraséologie (Riffaterre). Sur la scène de l'analyse textuelle, l'intertextualité prend alors toute la place disponible, en particulier au détriment de la notion d'auteur. Il n'y a pas de littérarité en dehors d'elle ${ }^{4}$; il n'y a pas non plus de texte en dehors d'elle ${ }^{5}$. Cette période est le lieu d'une dilatation extrême de la notion d'intertextualité: investigation nécessaire, mais qui ne pouvait se prolonger, car si tout est intertextualité, la notion risquait de devenir inutilisable.

La troisième période est celle du resserrement. L'effort de définition fourni par G. Genette apporte une clarification qui, depuis, n'a cessé de faire l'objet d'un large consensus. Selon Genette, il convient de distinguer une intertextualité au sens étroit, définie par la «relation de coprésence entre deux ou plusieurs textes», c'est-à-dire la "présence effective d'un texte dans un autre $»^{6}$. Son repérage se base sur l'étude de passages courts dans lesquels un emprunt est identifiable; on utilise les expressions «texte citant» pour le second texte et «texte cité» pour le texte préexistant, même si la «citation» n'est pas la seule forme d'emprunt possible. Incluant la notion précédente, il se présente, par ailleurs, une intertextualité au sens large pour laquelle Genette emploie le terme de «transtextualité», mais que la communauté a continué à nommer «intertextualité»: elle est «tout ce qui met [le texte] en relation, manifeste ou secrète, avec d'autres textes $»^{7}$. La mise en relation peut concerner l'œuvre entière et son support peut se manifester sous forme d'unités discontinues, comme un motif narratif, une structure ou un style d'écriture.

La question de la relation entre intertextualité et traduction a été réduite par Genette à une relation d'inclusion: parmi les divers types de relations intertextuelles au sens large figure la relation hypertextuelle qui unit deux textes tels que l'un (hypertexte) est dérivé d'un autre (hypotexte) qui lui est antérieur. La traduction, d'après cette définition, est une forme d'hypertextualité, mais il en est bien d'autres.

Pour les traductologues, les choses sont moins simples. Chez Berman, l'hypertextualité est réinterprétée comme l'un des modes de la traduction, mode qu'il rejette au même titre que la traduction "ethnocentriste ${ }^{8}$. Le rapport entre polyphonie et traduction a été très largement étudié par Barbara Folkart ${ }^{9}$, dans la double perspective de la traduction du discours rapporté et de la traduction comme discours rapporté. Cette double perspective sera aussi la nôtre; cependant, le champ d'étude que nous proposons ici est plus restreint, puisque la définition de l'intertextualité nous limite au cas où le discours «rapporté» est un discours attesté à l'extérieur du texte qui le 
convoque, et préexistant à lui. Par ailleurs, l'intertextualité fait appel à des techniques qui ne sont qu'en partie les mêmes que celles du discours rapporté. Enfin, si nous rejoignons cet auteur sur la conclusion de l'autonomie du texte traduit, nous chercherons à établir celle-ci moins en considérant les modalités de la ré-énonciation traductive qu'en analysant celles de la réception du texte traduit.

\section{Les enjeux de l'intertextualité}

Si tout texte comporte peu ou prou une composante intertextuelle, l'existence d'une pratique de réexpression du texte dans une autre langue doit forcément poser le problème de la relation entre intertextualité et traduction. Mais il importe de montrer précisément, exemples à l'appui, quels aspects du texte original sont concernés par l'intertextualité. Il s'avèrera que l'intertextualité intervient très directement dans les différents paramètres constitutifs du sens et de l'effet, qui conditionnent le travail du traducteur. Les exemples utilisés seront principalement empruntés à la littérature allemande de la fin du $\mathrm{XIX}^{\mathrm{e}}$ et du $\mathrm{Xx}^{\mathrm{e}}$ siècle, avec des traductions françaises et anglaises.

Tout d'abord, l'intertextualité donne accès à une partie de l'information qui peut ne pas s'exprimer autrement dans le texte. L'exemple d'Effi Briest, roman de Fontane paru en 1894, servira à le montrer. Effi Briest est une Emma Bovary prussienne, jeune et sensible, trop tôt et mal mariée à un équivalent de sous-préfet qui la sacrifie à sa carrière. Effi succombe aux tentatives de séduction du major Crampas, ami et collaborateur de son mari. Dans ce monde oppressant et oppressif qu'est la société prussienne des années 1880, l'aventure entraînera, à terme, le déshonneur et la mort des deux protagonistes. La scène de séduction décisive, au chapitre 17, se déroule au bord de la mer du Nord et les déclarations utilisent de façon détournée une poésie amoureuse récente, celle de Heine et de Goethe. Les emprunts intertextuels contribuent à caractériser les personnages: le beau parleur qui sait utiliser cet atout social qu'est la culture littéraire, la jeune aristocrate élevée à la campagne qui joue subtilement d'une ignorance vraie ou feinte de celle-ci, mais qui connaît la culture populaire. Ils contribuent aussi à caractériser ce monde prussien, si peu romantique mais où les écrivains jouissent d'un grand prestige, monde d'interdits où le désir ne peut s'exprimer qu'à mots couverts. La présence intertextuelle délivre donc une part importante du sens du texte.

L'intertextualité contribue aussi à façonner la voix du narrateur. Avec la simple instruction, donnée au lecteur par des emprunts répétés, de se remémorer la poésie de la tradition romantique, Fontane, romancier discret et très pudique, réussit, sans paraître s'y mêler lui-même, à suggérer la naissance, entre les deux personnages, d'une relation d'un érotisme très tendu qui est l'une des grandes réussites de ce chapitre et n'est sans doute pas pour rien dans la modernité d'Effi Briest. Par ailleurs, en empruntant les mots de Heine, le texte livre tout un commentaire sur le destin d'Effi et donne des signes d'une issue probable. Ce commentaire peut se faire lors même que le narrateur s'interdit toute incursion explicite: le roman est tissé de renvois, d'indices, de signaux, de symboles, grâce auxquels le récit, tout en se déroulant, signale son sens. Ainsi, la présence intertextuelle contribue à créer ce ton très particulier que le lecteur ressent comme la voix du romancier Fontane.

L'intertextualité conditionne également cette relation entre le texte et son lecteur implicite qui constitue l'effet. Selon les analyses un peu circulaires, mais cependant 
éclairantes, de W. Iser ${ }^{10}$, l'effet est le résultat de l'interaction entre les structures du texte, destinées à jouer d'une certaine façon sur la sensibilité du lecteur, et les caractéristiques cognitives et émotionnelles de ce lecteur, qui est lui-même inscrit dans les structures du texte: lecteur implicite, donc. Dans le roman étudié, l'effet «érotique» présuppose chez le lecteur la connaissance de la poésie de Heine. Au-delà de ce premier effet, le texte vise donc une seconde réaction: la perception d'une appartenance commune, d'une complicité culturelle entre la voix qui s'y exprime et le lecteurimplicite - auquel elle s'adresse. Les lecteurs berlinois des années 1880, qui composèrent le premier lectorat réel de Fontane, étaient naturellement prêts à se couler dans ce rôle, donc à ressentir les effets prévus par le texte. Pour le lecteur allemand contemporain, l'identification, variable selon sa compétence culturelle, reste généralement facile et contribue à assurer l'un des plaisirs de cette lecture.

Enfin, et peut-être surtout, l'intertextualité donne accès à ce que l'auteur veut dire à travers l'histoire qu'il raconte. Effi Briest, malgré tout l'attachement de Fontane à la Prusse, est un plaidoyer pour une société plus ouverte et qui méconnaîtrait moins rudement les droits et les sentiments naturels de l'individu, une société qui serait en état de citer Heine sans oublier hypocritement le sens progressiste et contestataire du message de celui-ci. Une autre œuvre narrative, Amok, de S. Zweig, paru en 1922, met en scène un médecin des colonies au début du $\mathrm{xx}^{\mathrm{e}}$ siècle, homme en révolte contre son existence, contre la déontologie de son métier et la culture humaniste traditionnelle. Il raconte son histoire à un héros-narrateur, touriste et poète, qui est, lui, très en phase avec cette culture. L'opposition se concrétise autour de différents types de renvois intertextuels. Loin de se limiter à être l'arrière-plan d'une anecdote, ce conflit entre «le monde d'hier» et la réalité du $\mathrm{xx}^{\mathrm{e}}$ siècle est bien, on le sait, celui qui a traversé l'œuvre et déchiré la vie de Stefan Zweig. Par textes interposés il y a donc, dans chacun de ces récits, l'affrontement de deux visions du monde - Barthes dirait: de deux idéologies -, affrontement dont l'issue suggère la visée de l'auteur.

Il n'a été question ici que de textes narratifs. Mais de façon analogue, les caractéristiques particulières à d'autres variétés de textes, humoristique, ironique ou polémique, correspondent souvent à un certain traitement de l'intertextualité. Plus largement, si c'est dans le domaine littéraire que l'intertextualité a été le plus étudiée, le phénomène concerne également les domaines non littéraires: un coup d'œil dans la presse en apporte facilement la preuve. Il est également présent dans ces «textes» particuliers que sont les échanges de la vie quotidienne. À travers les extraits de romans analysés ici, c'est donc de textualité en général qu'il sera question et non de littérarité.

\section{Traduire l'intertextualité}

Si l'intertextualité intervient pour conditionner différents aspects du sens et de l'effet communiqués par le texte, on peut facilement imaginer que, dans le cas d'une lecture différée et, plus encore, dans celui d'une traduction, la réception du texte par un lecteur réel éloigné des caractéristiques du lecteur implicite va changer notablement les conditions du jeu intertextuel. Est-il possible de traduire un texte sachant qu'une partie du sens et de l'effet se construit en fonction d'autres textes? Si «l'intertextualité parle une langue dont le vocabulaire est la somme des textes existants ${ }^{11}$ », peut-on traduire un texte écrit dans cette langue, dont le dictionnaire est toute une bibliothèque? 
La question ne comporte pas de réponse simple: elle suggère plutôt de rechercher les différents facteurs qui rendent l'opération facile ou difficile, voire possible ou impossible.

\section{A. Facteurs relatifs au texte citant}

Pour délimiter et décrire, dans le texte original, les lieux d'intertextualité, il convient de se munir de quelques définitions, que nous emprunterons pour l'essentiel à Genette $^{12}$. On appellera «trace intertextuelle» une séquence du texte qui peut être reconnue comme provenant d'un autre texte. Cette réapparition peut être littérale, le retour du déjà dit ne comportant aucune transformation du signifiant d'origine; au contraire le signifiant d'origine peut être transformé, tout en restant identifiable. Par ailleurs, le réemploi peut être déclaré: la séquence empruntée est alors accompagnée d'une ou plusieurs marques explicites de son origine allographe (nom d'auteur, titre d'œuvre, guillemets, etc.). Il peut ne pas l'être: Genette utilise dans ce cas le terme de «plagiat», fâcheusement moralisant. En croisant ces deux axes, on voit se dessiner quatre types de traces intertextuelles, qui seront présentées à l'aide d'exemples empruntés aux deux romans allemands déjà évoqués; chaque extrait de texte original est donné ici avec des traductions, mais ces dernières ne seront analysées qu'ultérieurement.

La citation est un emprunt littéral et déclaré.

\section{Exemple 1: Effi Briest}

C’est Crampas qui parle...: «(Seegespenst ist) etwas länger als „Du hast Diamanten und Perlen“ oder „Deine weichen Lilienfinger“", und er berührte leise ihre Hand. (153)

Traduction A: "(Fantôme marin» est) à peine plus long que «Les lis blancs de tes doigts» (et il lui toucha légèrement la main). (164)

Traduction B: («Fantôme des mers» est) un peu plus long que «Tu as des diamants et des perles...» ou «Tes doigts de lys si délicats...* ${ }^{*}$. Crampas effleura doucement sa main. (685)

Note: all.: deine weichen Lilienfinger. Intentionnellement, Crampas remplace le weissen («blancs») par weichen; est-ce pour ôter au lys un peu de sa blancheur virginale, ou plutôt pour trouver un prétexte à sa caresse? Les deux, peut-être.

Traduction C: «("Sea Spectre" is) a bit longer than "You have diamonds and pearls" or "Fingers soft and lily-white" ... and he gently touched her hand.» (100)

Note (sans appel de note): "You have diamonds", "Fingers soft...". First lines of poems from Heine's collection Buch des Lieder (1827). The word "soft" has been added by Crampas.

Dans cet exemple, trois poèmes de Heine sont convoqués dans l'esprit du lecteur, l'un par son titre, les deux autres (qui n'ont pas de titre) par leur premier vers. Ces deux vers font l'objet d'une citation: présentation littérale, signalée par les guillemets et, un peu plus haut dans le texte, par le nom de l'auteur. Que l'une des deux citations soit volontairement inexacte pose d'autres problèmes, qui ne seront pas abordés ici.

La référence est un emprunt non littéral mais déclaré.

\section{Exemple 2: Effi Briest}

C'est Effi qui parle...: «Wenn ich Sie recht verstehe [...], so haben Sie vor, sich vor der Zeit auf den König von Thule hin auszuspielen. [...] Die Rolle, die Sie mir dabei zudik- 
tieren, ist mir zu wenig schmeichelhaft. Ich mag nicht als Reimwort auf Ihren König von Thule herumlaufen.» (158)

A: «Si je vous comprends bien [...] vous avez l'intention de jouer avant l'heure le rôle du roi de Thulé. [...] Seulement (le rôle) que vous me réservez est trop peu flatteur pour moi.» (168)

$\mathrm{B}:$ «Si je vous comprends bien [...] vous avez l'intention de jouer à vous prendre, avant terme, pour le roi de Thulé* [...] Le rôle qu'en la circonstance vous m'imposez à moi est trop peu flatteur à mon goût. Je n'ai pas l'intention de faire figure de rime avec votre roi de Thulé.*» (689)

Note 1: Effi provoque Crampas doublement. C'est elle qui lance dans la conversation le thème du roi de Thulé. Et puis «avant terme» semble bien anticiper sur les événements.

Note 2: La rime en question (sur Thule) est «Buhle»: «amante, maitresse».

C: «If I understand you correctly, [...] you see yourself as the King of Ultima Thule, long before your time. [...] But I have to say that the role you see fit to cast me in is too unflatering. I don't want to run around as the rhyming appendage of the King of Thule.» (103)

Note: «King of Ultima Thule». In Goethe's ballad Der König in Thule the king is given a goblet by his «mistress» (Buhle= rhyming appendage) as she dies.

Dans la première partie de cet exemple, la ballade de Goethe Der König in Thule (1774) est évoquée par l'intermédiaire du nom du personnage principal, «der König von Thule», qui constitue une référence. On considérera comme référence toute indication permettant d'identifier un texte et de le rendre présent à l'esprit du lecteur, mais sans le citer littéralement: titre, nom d'auteur, résumé, nom de personnage ou de lieu, paraphrase, indication bibliographique, etc.

L'allusion est un emprunt non littéral et non déclaré.

Dans la deuxième partie de l'exemple précédent, la séquence «Reimwort auf [...] Thule», est une sorte d'énigme dont le lecteur, aidé par la référence, trouve la solution dans le texte du poème évoqué: le mot «Buhle». Ainsi le contenu même de l'allusion reste non-dit: l'allusion est une citation en blanc. Comme l'allusion grivoise ou malveillante de la conversation courante, elle tire son efficacité toute particulière d'être un signifiant présent et absent, son absence étant suffisante pour innocenter le locuteur et sa présence, susceptible de compromettre le destinataire.

Pour éviter «plagiat», nous parlerons pour le quatrième type d'emprunt (littéral) non déclaré.

\section{Exemple 3: Amok}

C’est le médecin qui parle...: Muß der wirklich sein Leben hinwerfen und sich Wasser ins Blut schütten, wenn [...] irgendeiner kommt und will, daß er edel sei, hilfreich und gut? (15)

A: Faut-il que, vraiment, il sacrifie sa vie et se tourne les sangs [...] quand un homme vient lui demander d'être noble, secourable et bon? (26)

B: Faut-il que, vraiment, il sacrifie sa vie et se tourne les sangs [...] quand un homme vient lui demander d'être noble, secourable et bon*? (38)

Note: Effet de quasi-citation dans le texte allemand. «Edel sei der Mensch, hilfreich und gut» est en effet le premier vers d'un célèbre poème de Goethe intitulé Das Göttliche (Le Divin). 
Dans ce passage qui ne comprend aucune marque de citation, le lecteur comprend que l'apparition des trois adjectifs «edel, hilfreich und gut», disposés dans cet ordre, a peu de chance d'être le fait du hasard; il identifie donc une citation non déclarée, évoquant tout un poème qui est l'un des grands standards de l'idéalisme classique.

Face à la traduction, ces quatre types de traces ont des comportements relativement différents. Pour le montrer, nous allons revenir sur les traductions citées pour les exemples précédents.

La traduction d'une référence se ramène souvent à la transcription de noms propres, en respectant, s'il y a lieu, l'usage de la langue d'arrivée, comme ici dans l'exemple 2.

La traduction d'une citation, comme dans l'exemple 1, pose un problème plus complexe. Elle doit respecter non seulement le sens du fragment cité, mais l'effet qu'il produit (ici: une vibration poétique) qui justifie qu'il puisse faire l'objet d'une citation et non d'une simple référence. Or il faut parfois de la bonne volonté au lecteur pour être sensible à la suggestion d'une citation traduite: «Les lis blancs de tes doigts» ne vibre pas beaucoup, pas plus que «Tes doigts de lys si délicats...». Il est vrai que la séquence originale n'a, elle non plus, rien de très remarquable, mais elle a derrière elle tout un poème et toute une poétique. C'est donc moins la qualité intrinsèque de la citation qui constitue son efficacité que son contexte, littéralement absent mais présent à l'esprit du lecteur par le fonctionnement même du renvoi. La citation traduite, quant à elle, n'a souvent aucun contexte sinon simulé, elle est pur trompe-l'œil et ne peut donc se permettre d'être stylistiquement déficiente. Certains traducteurs résolvent parfois le problème de la traduction de la citation en passant en mode non littéral, c'est-à-dire en remplaçant la citation par une paraphrase, assortie ou non d'une référence.

L'allusion présente pour le traducteur plusieurs types de difficultés qui peuvent se superposer. Dans l'exemple 2, le texte évoqué est indiqué en clair par la référence qui accompagne l'allusion, mais ce n'est pas toujours le cas. Même en présence d'une référence, le texte qui contient la solution de l'énigme n'est pas littéralement présent dans le texte citant et ne peut donc pas être traduit. Enfin, dans le cas envisagé, le mot «en blanc» n'est désigné que grâce à un jeu sur le signifiant de la langue originale (la rime), jeu qui ne peut être conservé par la traduction. Le traducteur A a purement et simplement supprimé le passage. En B et C, on trouve dans le texte des traductions relativement littérales qui ne seraient pas compréhensibles sans la note explicative présente en bas de page ou en fin de volume. Il y a donc dispersion du sens sur deux lieux, dont l'un prend en charge la trace explicite, tandis que l'autre explicite le non-dit.

La traduction d'un emprunt non déclaré (exemple 3), dans la mesure où il est non déclaré, semble comporter moins de contraintes. La traduction A se contente de restituer le simple sémantisme de la trace, mais c'est au prix d'une amputation, puisque le renvoi, et donc l'arrière-plan d'un conflit d'idéologies, est perdu. En B, le réviseur a ajouté une note pour signaler la présence de l'emprunt. Mais la teneur du texte cité n'y apparaît pas, et la compréhension ne peut, au mieux, qu'être le résultat d'une inférence sur le titre du poème: le conflit d'idéologies ne peut être reconnu que par un lecteur déjà informé.

Les difficultés de traduction et les méthodes employées pour les résoudre sont donc variables selon la forme de la trace. Un autre type de problème concerne non plus la trace elle-même, mais son mode d'intégration dans le contexte du texte citant. 
La trace intertextuelle, quelle que soit sa nature, s'intègre parfois, dans la phrase ou le passage qui la contient, sans créer de perturbation majeure au niveau du sémantisme: c'est le cas des exemples 1 et 3 . Dans ce cas, une traduction relativement proche du texte original, à défaut de restituer le sens intégral, est du moins compréhensible. Mais la trace peut aussi s'insérer de façon telle que seul le recours au texte cité permette de construire un sens satisfaisant pour l'ensemble du passage citant. Sans ce recours, le passage est inintelligible: selon Genette, il est «non recevable ${ }^{13}$ et Riffaterre parle d'une anomalie sémantique qu'il appelle «agrammaticalité» ${ }^{14}$; pour l'un et l'autre, cet accident sémantique est le signe le plus fréquent de la présence d'une allusion. La deuxième moitié du corpus 2 en est un exemple. Ce genre de passage est un défi au traducteur, dans la mesure où il interdit une traduction au niveau du simple sémantisme. Le sens ne peut être rendu que par une solution qui prendrait en compte à la fois le texte citant et le texte cité et qui restituerait conjointement le sens et l'effet: seule une libre recréation du traducteur pourrait relever le défi. En l'absence d'inspiration, il reste comme issue la suppression pure et simple du passage, comme en $\mathrm{A}$, ou la besogneuse note explicative, comme en B et C.

\section{B. Facteurs relatifs au texte cité}

Facilité ou difficulté à traduire un passage intertextuel ne dépendent pas seulement du texte citant, mais aussi du texte cité. Dans la problématique de l'intertextualité, l'expression «texte cité» doit être comprise au sens large: texte écrit, mais parfois texte non écrit (savoir de type scientifique, discours social, sagesse populaire, discours idéologique) ou système signifiant non verbal (iconographie, musique). Ici, les exemples choisis sont tels qu'on en restera cependant au cas classique où le texte cité est effectivement un texte écrit.

Le texte cité intervient dans le sens du texte citant pour beaucoup plus que le seul fragment emprunté, dont le «volume» effectivement présent peut être très faible. Quoi qu'il en soit, ce volume est toujours peu de choses par rapport à ce qui est convoqué dans l'esprit du lecteur: un passage entier, une œuvre, l'œuvre complète d'un auteur ou tout le corpus des textes d'une époque. Par l'intermédiaire du fragment, c'est le tout dont il est détaché qui participe ainsi, sur le mode de la synecdoque, à la constitution du sens du texte citant. Pour un texte citant moyennement riche, c'est donc bien déjà toute une «bibliothèque» qui est mobilisée. Par ailleurs, la contribution du texte cité au sens du texte citant n'est jamais un simple ajout, la mise en relation des deux textes ayant déjà par elle-même une fonction sémiotique: L. Jenny ${ }^{15}$ distingue une intertextualité «faible», qui peut être simplement la revendication d'un ancrage dans un auteur, une culture ou une idéologie, et un véritable conflit, le texte cité étant alors considéré comme représentant d'une pensée, d'une poétique ou d'une idéologie surannée, pesante ou dangereuse: conflit dont le texte cité ne sort pas indemne et dont l'issue constitue le véritable enjeu, la visée du texte citant. Pour M. Riffaterre ${ }^{16}$, plus radical, il n'y a intertextualité que lorsque ce conflit entre «codes antagonistes » peut être repéré.

Grâce à ce mode de fonctionnement, l'intertextualité permet une gestion très économique du sens: le texte produit un maximum de sens avec un minimum de signifiant. Mais, pour ce faire, elle suppose la coopération du lecteur: le sens du texte citant ne lui sera donné, l'effet ne lui sera sensible que dans la mesure où il sera 
lui-même en état d'apporter la connaissance du texte cité. Il faut donc faire intervenir la notion de «compétence intertextuelle» du lecteur réel, celle-ci étant définie comme la connaissance, antérieure à la lecture du texte citant, des textes cités et des moyens habituels d'y faire référence. Si nous transposons au cas des «sujets lisants» l'analyse des compétences des «sujets parlants» proposée par C. Kerbrat-Orecchioni, nous dirons que ces deux aspects ressortissent aux deux catégories appelées par cet auteur «compétence encyclopédique» et «compétence rhétorico-pragmatique» ${ }^{17}$. Lorsque le lecteur réel n'est pas très différent du lecteur implicite, cette coopération se fait bien. Mais qu'en est-il dans le cas du texte traduit, reçu par un lecteur ayant un tout autre univers de référence et disposant donc d'une tout autre compétence? Quand on traduit un texte, on ne traduit pas en même temps sa «bibliothèque»; une fois traduit, le texte peut donc se retrouver très loin d'elle: décontextualisé. Euvre par œuvre, mais aussi passage par passage, le travail du traducteur sera conditionné par le degré de cette décontextualisation, c'est-à-dire par la distance faible, moyenne ou immense, entre culture d'accueil et texte cité.

La décontextualisation est faible lorsque la présence culturelle du texte cité est identique ou analogue dans les deux cultures mises en contact par la traduction; c'est le cas de cultures proches ayant des zones de références communes. Un emprunt à la Bible, par exemple, pose généralement peu de problèmes lors d'une traduction entre langues européennes, chacune des cultures correspondantes ayant, malgré des nuances, un même ancrage dans le texte sacré; dans chacune, une version de la Bible dans la langue nationale peut être considérée comme familière au lecteur. Ainsi, la traduction d'un emprunt biblique se ramène souvent à une simple recherche de correspondance.

La décontextualisation est moyenne quand le texte cité est déjà traduit et plus ou moins familier dans la culture d'accueil. La ballade du roi de Thulé (exemple 2) est connue en France car Goethe y est depuis longtemps traduit (Nerval, 1828); mais c'est grâce aux opéras (Berlioz, 1846, Gounod, 1859) qu'elle s'est trouvée intégrée de façon particulièrement efficace dans la culture française. Le lecteur français disposant d'une compétence encyclopédique moyenne n'est donc pas déconcerté; le lecteur anglais, en revanche, semble avoir besoin d'une note. Quant à Heine, il a, lui aussi, été traduit en français de bonne heure, et toute la partie de sa vie passée à Paris a fait de lui l'un des poètes allemands les plus familiers, au moins de nom, au lectorat français. Pourtant, les renvois à Heine ne "passent» pas très bien, car le Livre des Chants n'est pas suffisamment intégré pour que les poèmes évoqués soient reconnus par le lecteur français.

La décontextualisation est à son maximum lorsque, dans la culture d'accueil, la connaissance du texte cité peut être considérée comme nulle. Le pavillon d'Or de Mishima raconte l'histoire d'un jeune bonze appartenant comme son auteur à la "génération de la défaite», en révolte contre la culture japonaise traditionnelle, sa spiritualité et son esthétique. Dans le texte original, ancrage et révolte s'articulent autour de la présence de nombreuses citations; selon le traducteur, «il est un (Japon) qu'on a plaisir à retrouver dans le roman de M. Mishima: c'est le Japon lettré, héritier respectueux d'une riche culture. Il arrive sans doute que les citations aient quelque chose d'agressif et, parfois même, agacent. Au moins sont-elles bien choisies, et d'un effet sûr.» ...et de commenter quelques-unes de ces citations. Mais, dans le cours de sa lecture, et même lorsque le texte est brièvement éclairé par une note, le lecteur non spécialiste n'est pas en mesure d'éprouver ce plaisir ni de ressentir cette agression, il n'est pas en 
mesure non plus d'estimer le poids culturel et idéologique du texte cité, donc de construire le sens produit par la citation dans le texte citant. C'est tout un pan de l'œuvre, peut-être l'essentiel, qui lui échappe.

Comment les problèmes traductifs posés par la décontextualisation sont-ils résolus? Généralement par une aide du traducteur, qui remet le texte cité à la portée du nouveau lecteur: notes en bas de page ou en fin de volume, glossaire, introduction ou postface, la traduction s'entoure de tout un métatexte critique qui devient le paratexte du texte traduit. La note peut, tout en restant discrète, apporter le complément de sens pertinent (exemple $1, \mathrm{C}$ ou exemple $2, \mathrm{~B}$, deuxième note). Mais la note est un exercice difficile, n'allant pas toujours jusqu'au point où elle deviendrait vraiment éclairante (exemple 3, B) ou substituant à l'information attendue un commentaire qu'il appartiendrait au lecteur de faire ou de ne pas faire (exemple 1, B ou 2, B, première note). Par ailleurs, même lorsqu'elle est inévitable et pertinente, l'intervention du traducteur modifie de façon essentielle les conditions de lecture de l'intertextualité:

Le propre de l'intertextualité est d'introduire à un nouveau mode de lecture qui fait éclater la linéarité du texte. Chaque référence intertextuelle est le lieu d'une alternative: ou bien poursuivre la lecture en ne voyant là qu'un fragment comme un autre, qui fait partie intégrante de la syntagmatique du texte - ou bien retourner vers le texte-origine [...] C'est simultanément qu'opèrent ces deux processus dans la lecture - et dans la parole - intertextuelle, étoilant le texte de bifurcations qui en ouvrent peu à peu l'espace sémantique $^{18}$.

Alors que, dans la langue originale, le fragment porteur d'intertextualité ouvre deux processus possibles de lecture, mais correspondant à une seule formulation, parce qu'il exploite à cet endroit précis une connaissance qu'il est inutile d'expliciter, la traduction amène souvent à séparer ces deux lectures, affectant l'une au texte et l'autre au paratexte: la "bifurcation» devient détour, le «simultanément» se perd, et le texte «étoilé» devient édition savante.

D’un point de vue théorique, une autre solution au problème de la décontextualisation doit être mentionnée: il s'agirait de remplacer le texte cité par un autre, plus proche du lecteur second et censé produire le même effet de sens. Ainsi, Marguerite Yourcenar, traduisant V. Woolf, propose de traduire "certaines phrases contenant une allusion à des thèmes ou images de la poésie anglaise [...] en tâchant d'obtenir de mêmes effets avec des thèmes analogues connus du lecteur français ${ }^{19}{ }^{\prime}$. U. Eco, quant à lui, impose:

Dans Le pendule de Foucault, j'essaie de montrer l'incapacité d'un de mes personnages à regarder le monde sinon à travers des citations littéraires. Or, dans un des chapitres, une description de paysage faite par ce personnage fait référence à une phrase célèbre dans le répertoire littéraire italien mais inconnue des lecteurs français qui, par là même, ne peuvent saisir mon dessein. J'ai donc demandé à mes traducteurs de transformer radicalement le texte source en y introduisant une référence à Lamartine afin que l'allusion littéraire soit assimilée par le lecteur français ${ }^{20}$.

Une telle suggestion peut retenir l'attention lorsqu'elle se présente sous une grande signature, à plus forte raison lorsqu'elle provient de l'auteur du texte. Pour le reste, considérons provisoirement que l'adaptation est trop éloignée des goûts actuels pour pouvoir être retenue. 


\section{Facteurs «humains»}

À ces paramètres afférant au texte citant et au texte cité s'ajoutent ceux concernant les acteurs humains mis en jeu par le processus de communication: le traducteur et son lecteur. Côté lecteur, la notion, précédemment utilisée, de «compétence intertextuelle» implique que le traducteur ne peut élaborer de stratégie sans connaître le niveau de compétence et, partant, le niveau d'exigence des lecteurs auxquels la traduction sera destinée: fixer le profil de ces derniers est généralement le rôle de l'éditeur. C'est surtout dans le cas de décontextualisation moyenne qu'il y a lieu de prendre en compte très précisément le lectorat envisagé. Ainsi, à une même époque, l'existence, pour une même œuvre, de plusieurs traductions réalisées avec des stratégies différentes pour des lectorats différents, est pleinement justifiée. Un traitement différencié dans la restitution de l'intertextualité n'a d'ailleurs rien de particulièrement choquant: il est d'autant plus normal que, dans la langue originale, la perception de l'intertextualité est également variable selon la compétence et l'histoire individuelle du lecteur ${ }^{21}$.

Côté traducteur, la traduction de l'intertextualité est souvent dépendante du rang occupé par celui-ci dans la chronologie des traductions de l'œuvre. Les traductions initiales sont celles où l'on constate le plus de pertes; les traductions largement «caviardées» n'y sont pas rares, comme le Berlin Alexanderplatz de Z. Motchane. Mais l'établissement de notes à l'occasion d'une seconde traduction ou d'une révision de la première n'est pas toujours une réussite, comme en témoigne la révision de Amok. $\mathrm{Au}$ niveau individuel, la traduction de l'intertextualité est l'un des domaines qui mettent le plus en jeu les qualités personnelles du traducteur, son habileté, sa culture, son sens de la langue. Dans ce domaine, la liberté et la créativité permettent parfois d'éclatantes réussites, que le théoricien ne peut que reconnaître et saluer sans en faire la théorie, car la liberté et la créativité sont, précisément, ce qui échappe à la théorie.

Peut-on donc traduire l'intertextualité? L'analyse précédente a mis en évidence, dans ce domaine, l'existence d'une «marge» de sens ou d'effet qui, dans les conditions normales (c'est-à-dire en excluant les cas faciles dus à une proximité des deux cultures et les réussites dues à un talent particulier du traducteur) semble échapper à la traduction. En effet, du fait de la décontextualisation, la conservation intégrale du sens découlant de l'insertion, sur le mode de la synecdoque, de tout un texte allographe ne paraît guère possible: le texte traduit n'utilise pas les ressources de la même langue, il n'évolue pas dans le même univers de référence, il n'est pas destiné au même lecteur. Une fois traduit, le texte peut signaler l'intertextualité de son texte directeur, il peut aussi l'expliciter, ou encore la simuler avec d'autres moyens, mais il ne peut pas à la fois conserver le même fonctionnement et produire le même effet. Il semble donc qu'il faille compter avec une certaine entropie, sans doute limitée par la technique, l'habileté ou la créativité du traducteur, mais qui, d'un point de vue théorique, ne peut être nulle.

\section{La traduction dans le champ intertextuel}

Si la traduction est décontextualisation, elle implique aussi une recontextualisation, installation du texte dans un nouvel environnement. Il faut donc examiner comment le texte traduit se comporte face aux composants du milieu qui le reçoit. Est-il exclusivement en relation avec son texte directeur ou peut-il entrer en communication avec 
d'autres textes? Est-il prêt à assumer la fonction de texte citant, voire à accepter le rôle de texte cité? Bref, est-il une entité autonome munie d'un statut intertextuel propre ou restera-t-il un clone?

\section{A. Un segment particulier: le titre}

Tenu par la fidélité à son texte directeur, il semble a priori exclu que le texte traduit s'arroge pour son propre compte la fonction de texte citant. C'est pourtant ce qui se passe de façon relativement fréquente, dans la traduction de cet élément un peu hors norme qu'est le titre. Hors norme, parce que sa fonction est plus importante que son sens. Il est en effet le premier vecteur de communication entre le texte et le lecteur: il doit, comme on dit, «accrocher» le lecteur potentiel. L'œuvre une fois lue, il restera son nom propre, la façon pratique dont on la désignera dans la communication de lecteur à lecteur. Le titre est donc une interface essentielle du point de vue de la réception et de l'édition; il a, avant tout, une fonction pragmatique ${ }^{22}$.

Une typologie des titres montre qu'il y a souvent, dans cette vitrine, une présence intertextuelle, qui revendique un arrière-plan culturel, déclare une fidélité ou annonce une contestation. Ainsi, "Also sprach Zarathustra (titre de l'œuvre parue en 18831885) affiche, dans le nom du réformateur iranien, la référence à un épisode de la réflexion religieuse et morale de l'Antiquité; il contient aussi un emprunt non déclaré à la Bible, sous la forme du segment «Also sprach... $»^{23}$. Ce titre annonce donc déjà la teneur du propos et sa visée polémique, le nouveau Zarathustra étant présenté comme celui qui, à l'instar de son père spirituel, va s'opposer à la nouvelle caste sacerdotale et à la nouvelle morale établie.

Le titre du roman de Günter Grass «Ein weites Feld» (1997) est un emprunt à Effi Briest: la formule favorite du père Briest, souriant et résigné, est présente, avec une très légère variation ( «ein weites/zu weites Feld»), à plusieurs reprises dans le récit de Fontane et y figure, entre autre, comme mot de la fin. Dans la bouche du personnage, la formule est un cliché, métaphore figée qui cite sans le déclarer le Urfaust de Goethe (v. 400: «das Feld ist gar zu weit», dit l'étudiant, découragé par l'ampleur du programme...). Chez Grass, la citation de Fontane est également non déclarée, mais dès la première page, le roman est tissé de références à cet auteur. Avec ce titre, Grass signale son goût tout particulier pour le romancier du siècle précédent; au-delà de ce lien affectif, il revendique pour sa propre œuvre une place dans une certaine réflexion sur l'Allemagne, à laquelle ont participé, avant lui, Fontane et Goethe.

Cet état de choses concernant les titres appelle, pour le traducteur, plusieurs questions distinctes: L'intertextualité manifestée dans un titre peut-elle être conservée pour le lecteur second? Si elle peut l'être, le titre ainsi traduit remplira-t-il encore la fonction pragmatique qui était celle de l'original? Sinon, que faire?

Pour des raisons déjà mentionnées, «Also sprach Zarathustra» est un cas techniquement facile: "Ainsi parlait Zarathoustra» a été trouvé dès la première traduction (H. Albert, 1901). Le titre français présente les mêmes caractéristiques intertextuelles que le titre allemand et se révèle, d'un point de vue pragmatique, tout aussi efficace. Il s'est imposé facilement, a contribué à la rapide fortune de l'œuvre en France et n'a été changé par aucun des traducteurs ultérieurs (M. Betz, G. Bianquis, M. Robert).

Les choses sont un peu plus difficiles pour «Ein weites Feld». Dans les traductions françaises publiées pour Effi Briest, la phrase du vieux Briest est traduite respectivement 
par «Il y a beaucoup de choses dans le monde» (traduction A) et «C'est un vaste champ de problèmes à méditer» ou "Un vaste champ de méditations » (traduction B). Aucune de ces formules n'est suffisamment prégnante pour qu'on puisse espérer qu'elle soit mémorisée par le lecteur; du reste, Effi Briest n'est pas assez intégré dans la culture française pour fonctionner facilement comme texte cité. La solution adoptée pour le titre de Grass, "Toute une histoire», remplace l'emprunt par une expression contenant un jeu de mot (histoire racontée/histoire des historiens) et renvoyant à un cliché ( «c'est toute une histoire» ou «il en a fait toute une histoire»), séquence verbale figée par l'usage, qui fait partie d'une phraséologie courante, mais sans renvoi à aucune œuvre particulière. Le double rebond contenu dans le titre allemand est donc perdu, ainsi que l'effet d'annonce voulu par Grass.

Quant à la traduction anglaise, ne pouvant, pour des raisons du même ordre, citer la traduction publiée pour Effi Briest ( «It's a vast subject»), elle installe le passage concerné en exergue - à la suite de l'épigraphe prévue par l'auteur - sous forme de citation en langue originale et référencée, puis propose une retraduction ad hoc ( «And Briest said quietly: "Oh, Luise, let it be... that takes us too far afield"»), qu'elle peut alors emprunter comme titre: «Too Far Afield». Ainsi sont résolus, au moins, le problème de la traduction de la trace et celui de l'identification du texte cité. Quant à la visée suggérée par le titre allemand, il n'est pas sûr qu'elle soit clairement identifiable dans l'univers de référence du lecteur anglophone.

Pour ces deux ouvres, les traducteurs ont simplement tenté, avec un succès variable, de conserver dans le titre traduit quelque chose de l'intertextualité présente dans le titre original. Mais il se trouve des cas plus singuliers où la traduction d'un titre s'émancipe. Le titre choisi par Proust pour désigner l'ensemble de sa grande œuvre, "À la recherche du temps perdu», ne semble pas mobiliser de renvoi intertextuel (sinon l'emprunt d'une phraséologie repérable, par exemple, dans « La recherche de l'absolu» de Balzac) et devrait être facile à traduire littéralement. Or la première traduction anglaise (Scott Moncrieff, 1922-1930) ne se satisfait pas de cette simplicité et propose: «Remembrance of Things Past», emprunt non déclaré au sonnet 33 de Shakespeare ( When to the sessions of sweet silence thought / I summon up remembrance of things past...»), qui doit être facilement identifié par le lecteur anglais. De ce fait peut-être, le titre s'est largement imposé comme la désignation anglaise de l'œuvre. Quant à la première traduction chinoise intégrale (1990), le titre choisi pour elle signifie littéralement «À la recherche des années passées comme l'eau qui s'écoule» ${ }^{24}$ : cette comparaison, qui tire son origine des Entretiens de Confucius, est une image courante dans la poésie chinoise traditionnelle.

Dans les deux cas, la traduction choisie pour le titre est un segment qui cite sans le déclarer un classique de la culture d'accueil: il y a donc, par choix délibéré du traducteur, une intertextualité propre au titre traduit. On peut, certes, critiquer la justesse de l'apport de sens ou du commentaire ainsi effectué: le sonnet de Shakespeare évoque un «bon vieux temps » qui n'est pas à l'horizon de Proust; quant à Confucius... Mais l'important est que, grâce à ce renvoi, le lecteur second puisse rattacher l'œuvre, au moins provisoirement, à une problématique et à une phraséologie de son propre univers de référence. La mobilisation d'un emprunt intertextuel originaire de la culture d'accueil est une recontextualisation de l'œuvre, qui promet au public éloigné un intérêt à lire le texte étranger et une certaine facilité à le faire: elle est une aide à l'intégration du texte et permet ainsi de sauvegarder la fonction pragmatique du titre. 
Il semble donc que, au moins dans ce cas particulier, une technique proche de ce que nous avons désigné ci-dessus comme «adaptation» conserve quelque légitimité, en permettant de réaliser une équivalence fonctionnelle.

Un cas encore plus curieux et plus complexe est celui de The way of all flesh, œuvre de Samuel Butler, écrite entre 1872 et 1884, publiée en 1903 après la mort de l'auteur. Dans ce roman en grande partie autobiographique, ce fils et petit-fils d'ecclésiastique attaque au nom de la science la tyrannie de l'idéologie chrétienne dans la société de son époque. Son titre désigne cette cible, avec un emprunt non déclaré à la Genèse ( God looked upon the earth, and, behold, it was corrupt, for all flesh had corrupted his way upon the earth», 6, 12). Le roman a été traduit en français en 1921. La traduction du titre aurait dû pouvoir se faire par simple correspondance («Dieu regarda la terre, et voici, elle était corrompue; car toute chair avait corrompu sa voie sur la terre $\left.{ }^{25} . »\right)$, mais une homophonie malencontreuse («voie»/ «voix»), une hésitation sur l'usage (certaines traductions de la Genèse disent «les voies») ont perturbé cette simplicité, provoquant la perplexité du traducteur, Valéry Larbaud, qui raconte:

Léon-Paul Fargue est en partie responsable de la traduction du titre du roman de S. Butler, The way of all flesh: «Ainsi va toute chair». Il m'a aidé à la trouver. Je lui en avais expliqué le sens, allant du mot à mot: La voie de toute chair («Et toute chair avait corrompu ses voies ", dit la Genèse) à: Comme se comporte toute chair, comme fait toute chair... Après un moment de réflexion, il dit simplement: «Zarathoustra?...» Le problème était résolu ${ }^{26}$.

Nous assistons ici en direct à la création d'une belle réussite. Le titre français conserve le renvoi au texte cité par le titre anglais, avec l'expression "toute chair», présente dans la Bible française; il redouble cet effet par l'emprunt de la tournure "Ainsi + verbe», biblique elle aussi. Mais cette tournure apporte avec elle une allusion totalement étrangère à l'original, puisque, en français, elle fonctionne aussi comme trace évoquant un «texte en blanc»: Zarathoustra. Cette circonstance singulière est instructive à deux égards. D'une part, concernant Butler, elle nous fournit un exemple supplémentaire d'intertextualité autonome dans une traduction. Ce renvoi à un élément qui, à cette époque, faisait partie de la bibliothèque de la culture d'accueil, a facilité l'accès du lectorat à l'œuvre nouvelle; de fait, le titre s'est immédiatement imposé. D'autre part, concernant Nietzsche, elle prouve - et la formulation de L.-P. Fargue le confirme - que, en 1921, le titre traduit «Ainsi parlait Zarathoustra» (ainsi que l'œuvre qu'il désigne) était suffisamment bien intégré dans la culture française pour devenir texte cité, et, qui plus est, sur le mode le plus difficile, celui de l'allusion.

De plus, le renvoi de Butler à Nietzsche n'est pas seulement une habileté stratégique; il se justifie totalement sur le plan du sens, avec le rapprochement de ces deux fils d'ecclésiastiques, contemporains, et ayant sans doute des raisons assez proches de se défier d'une société ancrée dans la religion. Du fait de ce renvoi, que rien dans l'original ne laissait prévoir - et pour cause -, on pourrait objecter que le titre traduit esquisse un commentaire sur l'œuvre, qu'il est donc de nature métatextuelle, et le critiquer au nom de la fidélité. Mais, dans la perspective qui est la nôtre, nous dirons plus volontiers que, par ce jeu d'échos, il se crée entre l'ancienne Perse, la Bible, le philosophe allemand et le romancier anglais un système inattendu de courts-circuits, où chaque texte enrichit la lecture des autres, réseau intertextuel, donc, qui - sans nul doute - fait sens. 


\section{B. L'œuvre entière}

Ce qui précède montre la nécessité d'étudier la présence du texte traduit dans le champ intertextuel au sens large, soit, comme nous l'avons dit, d'une façon qui permette de décrire les relations, «manifestes ou secrètes », qu'il établit en tant qu'œuvre entière avec d'autres œuvres présentes dans le champ qui l'accueille.

L'exemple choisi sera le premier chapitre de Der Verschollene (Amerika) de Kafka, texte d'abord destiné à être autonome sous le titre Der Heizer. C'est le récit du débarquement à New York du jeune Karl Rossmann, rejeté pour «faute» par sa famille, exilé sans horizon d'avenir, avec une valise pour seul patrimoine. Pendant la traversée, Karl a veillé jalousement sur cette dernière et sur les trésors qu'elle contient: un saucisson emballé par sa mère, un costume un peu rapiécé, quelques photos. Mais à l'arrivée, Karl oublie son parapluie en cabine et redescend dans le bateau, laissant sa valise à la garde d'un inconnu... Cette valise fait partie d'un «scénario» des plus banals - on embarque avec ses bagages, on les récupère à l'arrivée - permettant un habillage figuratif très classique pour assurer l'effet de réel dans le récit d'un voyage. Ici, cependant, apparaît un incident qui fait du scénario l'amorce d'un suspens: Karl va-t-il retrouver sa valise?

De l'aveu même de Kafka, cette «histoire de valise» est un emprunt, non déclaré dans le texte, mais référencé dans le Journal (8. 10. 1917). Dans le texte cité, David Copperfield, la valise est un tout petit détail perdu au milieu d'une foule d'autres éléments descriptifs. David, rejeté par son beau-père, quitte douloureusement sa mère pour se rendre en pension à Londres; en route, on change de véhicule: «The coach was in the yard, shining very much all over, but without any horses to it as yet; [...] I was $[\ldots]$ wondering what would ultimately become of my box $[\ldots]$ and also what would ultimatly become of me...» (page 56) Arrivé à Londres, David est attendu par un professeur de l'école qui l'emmène... sans la valise: "I was so ashamed to allude to a common-place thing like my box, to a scholar and a master at Salem House, that we had gone some little distance from the yard before I had the hardihood to mention it.» (page 63) Dickens aurait sans doute été bien étonné de savoir que ces quelques phrases recelaient une intertextualité potentielle... Nous apercevons ici le phénomène de rétroaction, normal dans le champ intertextuel, par lequel Der Heizer modifie notre lecture de David Copperfield.

Mais Kafka ne se contente pas d'emprunter à Dickens un détail réaliste: les phrases citées montrent déjà, chez David, l'identification du héros à sa valise, la peur de l'avenir exprimée par l'inquiétude concernant celle-ci, la timidité à défendre son seul bien face aux personnages d'autorité, que l'on retrouvera chez Karl. Kafka emprunte donc un ensemble déjà organisé de façon signifiante, c'est-à-dire un «motif». La réécriture de ce motif se fait sous le signe de l'hypertrophie et de la récurrence: la valise deviendra dans Der Heizer, puis dans Amerika, l'objet de nombreuses réflexions et de conversations sans fin, elle sera la manifestation toujours plus claire de la fragilité existentielle du héros, atteignant, de ce fait, un statut de symbole, ce qui est souvent le cas pour une variation hypertextuelle.

Le motif de la valise n'est pas le seul emprunt à David Copperfield. Kafka en signale d'autres, et les critiques en ont repéré davantage encore. Selon le passage du Journal, déjà mentionné, Le soutier serait une "pure imitation de Dickens» ("glatte Dickens-Nachahmung») dont l'auteur s'explique en ces termes: 
Comme je m'en aperçois maintenant, mon intention était d'écrire un roman à la Dickens, mais enrichi de tons plus vifs que j'aurais empruntés à mon époque et de tons plus mats que j'aurais mis de mon propre cru. Opulence de Dickens, il se laisse aller sans hésiter à une prodigalité extrême, cause de l'effroyable faiblesse de certains passages où, fatigué, il ne fait plus que brouiller les éléments qu'il a déjà. Impression barbare produite par cet ensemble extravagant, c'est là une barbarie que j'ai toutefois pu éviter, grâce à mon manque de vigueur et à ma qualité d'épigone ${ }^{27}$.

Malgré sa révérence liminaire, la relation de Kafka à Dickens est une relation de conflit: celui que provoque, entre deux écrivains, la différence de personnalité ( «opulence de Dickens» vs «mon manque de vigueur») et de style d'écriture ("prodigalité extrême» vs «barbarie que j'ai pu éviter»). De fait, la méthode de réécriture de Kafka procède en deux temps. Il s'agit d'abord d'annoncer une scène générique ou un motif présent dans l'hypotexte, qui doit créer l'attente d'une écriture conforme à ce dernier, puis de décevoir cette attente par une réalisation bien différente, provoquant un effet de frustration. Ainsi cet épisode, où une bande de matelots, ayant manifestement bien arrosé la fin de la traversée, fait irruption dans le salon du commandant, leur meneur s'exhibant dans une fort curieuse tenue, le tablier d'une fille de cuisine noué autour des reins... Au lecteur qui attendrait une scène à la manière de Dickens, avec sa verve, son abondance, son lexique coloré, Kafka oppose une description sèche, une syntaxe guindée et un lexique exsangue:

\section{Exemple 4a: Der Heizer}

Da bemerkte er die Mädchenschürze, riß sie herunter, warf sie zu Boden und rief: «Das ist ja ekelhaft, da haben sie mir eine Mädchenschürze umgebunden.» (42)

Traduction de A. Vialatte, 1946: Il aperçut le tablier de femme; il l'arracha, le jeta par terre et cria: «C'est dégoûtant; ils m'ont attaché un tablier de femme.»

Traduction de F. Mathieu, 2000: [...] «C'est vraiment dégoûtant, ils viennent de me mettre un tablier de femme.»

Si Kafka emprunte avec insistance les motifs de Dickens, tout en s'interdisant de manière systématique et assez ostentatoire les complaisances d'écriture qu'il dénonce chez son modèle, c'est cependant moins pour attirer l'attention sur le conflit de deux tempéraments d'écrivain que pour illustrer le conflit de deux époques, dont l'une est celle du paternalisme social et l'autre celle des débuts du capitalisme à l'américaine. En 1912, le destin du jeune homme déraciné, son incertitude existentielle relèvent d'une description moins haute en couleur, moins «bon enfant» qu'en 1849, ils nécessitent une évocation empruntant des «tons plus mats», plus « durs» («scharf», traduit ici par «vif»). Il s'agit d'alerter le lecteur face à la naissance d'un «monde industriel» destructeur des valeurs sociales (selon la thèse de W. Emrich ${ }^{28}$ ), à l'émergence d'un univers proliférant à l'infini où l'individu ne peut, comme Karl à la fin d'Amerika, que disparaître. Il y a donc, derrière les renvois répétés à l'hypotexte Dickens, toute une visée, dont il nous reste à examiner les avatars traductifs.

Chez la plupart des traducteurs français, depuis le premier (Vialatte, 1946) jusqu'au dernier (Mathieu, 2000), la banale «box» de David, devenue banale « Koffer» de Karl, se retrouve banale «malle» ou «valise» rien ici qui puisse étonner. Mais dans une autre traduction (Billman et Cellard, 1997), nous trouvons sur l'épaule du jeune homme un bien singulier "paquetage». Ce terme désigne, selon Le Robert, les «effets d'un soldat pliés et placés de manière réglementaire»: Mac Orlan n'est pas très loin; 
un peu plus, Karl aurait un «barda» et nous serions chez Céline. Cette traduction est loin d'être neutre: elle enrichit le contenu sémantique de la «valise» d'un trait absent chez Kafka. D’un point de vue microsémantique, ce «paquetage» est une infidélité.

Le sème supplémentaire qui vient d'apparaître se retrouve quelques pages plus loin dans notre second exemple. Là où les traductions citées travestissent le marin d'un «tablier de femme» microsémantiquement conforme au «Mädchenschürze» incolore et inodore de Kafka, celle de 1997 emploie une expression populaire qui sent la chambrée:

\section{Exemple $4 \mathrm{~b}$}

Traduction de Billman et Cellard, 1997: [...] C’est dégoûtant! Voilà qu'ils m’ont affublé d'un tablier de gonzesse! (112)

L'apparition du sème supplémentaire n'était donc pas un incident ponctuel: le lecteur, dès lors alerté, en découvre, régulièrement distribuées dans le texte, toute une série d'occurrences: «engrosser une bonne», «frapper comme un dingue», «zyeuter», «se tirer», "pêcher un beau gosse», «ficher le camp», « un freluquet», «souquer ferme», « un lamentable salmigondis», "les grouillots du navire», "mater cette tête de cochon», «de la fumisterie», «tenir la jambe», «s'en soucier comme d'une guigne», «bavasser», etc. La présence de cette isotopie, qui évoque le populaire, le familier, le rigolard, mais aussi le désabusé, le précaire, effectue un déplacement léger, mais global, un glissement vers le picaresque qui nous permet d'apercevoir, au-delà du texte de Kafka, le monde de Dickens.

En substituant ainsi un code à un autre, le traducteur prend à contre-pied la réécriture opérée par Kafka: il se rapproche de Dickens. La traduction s'installe donc, par rapport à ce qui était l'hypotexte de son directeur, dans une relation hypertextuelle toute différente. Au cas où l'on attendrait, au nom de la fidélité, que traduire consiste à dupliquer le position intertextuelle du texte directeur, il faudrait conclure à l'infidélité éclatante de cette traduction.

Or il est clair qu'une telle duplication, en admettant qu'elle fût souhaitable, est théoriquement et pratiquement irréalisable. Nous le montrerons en comparant les conditions de lecture du texte original et celles des deux traductions contemporaines.

En 1912, dans les pays de langue allemande, Dickens était très présent à l'horizon des lecteurs amateurs de romans. Pour le lecteur français contemporain, il est relativement effacé, sauf pour les spécialistes de littérature anglaise ou, dans un autre perspective, pour les «jeunes» lecteurs: un emprunt non déclaré à Dickens, qu’il soit emprunt d'un détail descriptif, d'un motif ou d'une scène générique, risque de passer inaperçu. Ainsi, Le soutier se trouve décontextualisé par rapport au texte cité par son original et le premier «mouvement» de la réécriture effectuée par Kafka a toutes les chances d'échouer. Aussi fidèlement qu'il tente d'imiter la méthode de Kafka, un texte français contemporain ne peut donc reproduire, par rapport au texte anglais de 1849, la relation mise en place par le texte allemand de 1912. Quant au second mouvement, il est promis au même destin. Car l'attente du lecteur français qui, en 1997 ou 2000, prend en main Amerika, n'est pas celle d'un divertissement picaresque et haut en couleur. Il sait qu'il va lire «du Kafka», avec sa «langue sobre», son univers «froid, triste, écrasant» (Le Robert 2) : cette vision est une doxa bien intégrée dans l'univers de référence français ${ }^{29}$. Une traduction qui, comme celle de 2000, emprunte des tons 
«mats» et «durs», un lexique pauvre, calqués sur ceux de Kafka ne produira qu'un effet de confirmation et non la frustration visée par l'original. Il est donc clair qu'une notion de la «fidélité» qui exigerait que le texte traduit duplique la position de son directeur dans le champ intertextuel, qu'il soit un alias de son original dans une autre langue, est nécessairement vouée à ne restituer ni l'effet ni la visée de celui-ci.

En revanche, la traduction peut tenter de recontextualiser le texte original d'une façon conforme à sa propre époque. En faisant apparaître explicitement dans le texte traduit une isotopie "dickensienne», en réveillant le souvenir de ce monde autrement que par un métatexte critique, le traducteur de 1997 rétablit à l'horizon du lecteur contemporain la présence d'un hypotexte qui, du fait de changements survenus dans l'univers de référence, avait cessé d'être actif. Il restaure donc l'effet de superposition produit par l'original kafkaïen. Il restaure également l'effet de frustration que nous avons décrit ci-dessus: abordant Le soutier, le lecteur contemporain qui s'attend à lire «du Kafka» ne peut pas ne pas être surpris, voire choqué ${ }^{30}$ de se voir servir «du Dickens». La traduction joue, pour se rapprocher de Dickens, sur des modifications stylistiques de même nature - mais de sens opposé - que celles dont Kafka s'était servi pour montrer qu'il s'en éloignait; elle transporte des conditions de 1912 à celles de 1997 ce jeu avec l'inversion de deux codes, cet effet de substitution inopinée de l'un à l'autre. Au lieu de se calquer sur son texte directeur, le texte traduit s'installe donc par rapport à lui dans un rapport intertextuel de même nature que celui qui existait entre le texte directeur et son propre hypotexte. De fait, il nous semble que la traduction étudiée ici exploite au mieux, dans l'esprit indiqué par Kafka, les nécessités qui lui sont imposées par l'univers de référence du lecteur français de 1997.

Ayant fait le choix de la recontextualisation, le texte traduit suggère une visée très proche de celle du texte original: ce jeune homme de famille modeste, perdu avec son seul «paquetage» dans un environnement étranger où chacun ne cherche qu'à l'instrumentaliser au service de son propre intérêt, dénonce en 1997, comme il le dénonçait en 1912, un monde où les valeurs morales sont perverties, l'identité menacée, l'avenir incertain. Le premier texte montrait, à l'aube du capitalisme, que ce monde était un monde d'émigrants; le second, rappelle qu'à l'aube de la globalisation il est un monde de migrants. Cette traduction fait découvrir un texte dont il apparaît qu'il a de nombreuses attaches avec le discours social de notre époque et avec sa littérature, littérature du déracinement et de la fragilité existentielle.

$\mathrm{Du}$ fait de cette homologie de moyens et de visée avec son texte directeur, Le soutier ne rentre pas dans les nombreuses catégories de textes dérivés analysées par Genette; il n'est pas non plus «hypertextuel» au sens de Berman. Il reste, par ce respect du sens, de l'effet et de la visée de son original, une «traduction»: nous rejoignons ici les notions d'auteurs qui nous sont proches, celle de «lien» (F. Israël), celle de «loyauté» (C. Nord), celle enfin d'«identité de signifiance»(J.-M. Gouanvic).

Ainsi, à la place de l'impensable «clonage», nous voyons se constituer une constellation intertextuelle qui est une relation triangulaire dans laquelle le texte traduit figure avec la même légitimité que les deux «originaux». De fait, étant le produit d'une énonciation autonome, parce qu'effectuée à un certain moment de l'histoire par un lecteur de cette époque et pour un lecteur de cette époque (nous rejoignons ici B. Folkart), le texte traduit est un original. Dans ce triangle, chacune des relation duelles est différente des deux autres. Le phénomène de rétroaction intertextuelle y 
fonctionne normalement, la traduction de 1997 modifiant notre lecture de Kafka ainsi que celle de Dickens ${ }^{31}$. Le texte traduit est donc bien un élément vivant qui travaille, comme les autres, dans le champ de l'intertextualité.

\section{Conclusion}

Au terme de ces réflexions sur le rapport entre intertextualité et traduction, il apparaît, d'une part, que le texte traduit ne peut pas proposer une réplique à l'identique de l'intertextualité de son texte directeur, mais qu'il occupe, dans un champ intertextuel forcément autre, une position forcément autonome. Il y a donc lieu d'opposer les traductions qui subissent la décontextualisation en essayant d'en contrecarrer les effets (mais c'est peine perdue) et celles qui jouent le jeu de la recontextualisation en acceptant d'entrer dans l'interaction, donc d'être un élément vivant. Ainsi, c'est probablement, en grande partie, le positionnement intertextuel d'une traduction qui détermine si cette traduction est vivante, lisible, ou si elle est désuète, inerte, périmée et parfois même périmée au moment même de sa parution ${ }^{32}$.

Nous voyons, d'autre part, que l'activité traductive occupe une place capitale au nombre des pratiques qui assurent la constitution du champ de l'intertextualité. En effet, au-delà des quelques années, décennies ou siècles où un texte peut être productif à l'intérieur de la culture-langue qui l'a vu naître, sa fécondité principale se déploiera à d'autres époques et dans d'autres cultures, donc par l'intermédiaire de ses traductions. Or, l'exemple de Kafka a montré que le texte traduit se trouve au centre d'un réseau intertextuel beaucoup plus complexe que celui de son texte directeur, puisqu'il se trouve, par la simple mécanique des choses, non seulement en relation avec son directeur et la «bibliothèque» de celui-ci, mais aussi avec les traductions précédentes du même original et avec la «bibliothèque» de sa propre culture. Quant à l'exemple de Butler, il a montré que, au-delà de ce champ minimum, le texte traduit se crée son champ intertextuel propre, y incluant d'autres textes, qui ne faisaient pas forcément partie du champ intertextuel de son directeur, mais qui, par effet rétroactif, y entreront. Le texte traduit, bien plus qu'un texte non traduit, contribue à enrichir le champ intertextuel de sa culture d'accueil. Ce sont les traductions qui créent des ponts entre les cultures du monde, qui densifient les réseaux déjà existants. C'est par leur jeu que se constitue cet ensemble constamment en train d'être réévalué, ce domaine en perpétuel mouvement, animé de glissements et de rebonds, de soubresauts et d'échos, cette immense toile frémissante qui est la bibliothèque de l'humanité.

\section{NOTES}

1. D. Maingueneau (1976).

2. P. Sollers (1968).

3. J. Kristeva (1969: 85).

4. Pour Riffaterre, «l'intertextualité est [...] le mécanisme propre de la lecture littéraire. [...] Chacun des mots qui composent [le texte] n'aura de fonction littéraire que lorsqu'il sera [...] compris [...] en fonction de l'intertexte qu'il présuppose.» (1979: 496)

5. Pour Barthes, «tout texte est un intertexte; d'autres textes sont présents en lui, à des niveaux variables, sous des formes plus ou moins reconnaissables: les textes de la culture antérieure et ceux de la culture environnante; tout texte est un tissu nouveau de citations révolues. Passent dans le texte, redistribués en lui, des morceaux de codes, des formules, des modèles rythmiques, des fragments de langages sociaux, etc., car il y a toujours du langage avant le texte et autour de lui.» (1973)

6. G. Genette (1982:8). 
7. id., 7.

8. A. Berman (1999: 39). Est hypertextuelle la traduction qui dépasse la «texture de l'original».

9. B. Folkart (1991). Signalons aussi, du même auteur, l'article "Métatextualité et traduction», Revue canadienne de littérature comparée, 1986, auquel nous n'avons pu avoir accès.

10. W. Iser (1985: 47-76).

11. L. Jenny (1976: 266).

12. G. Genette (1982).

13. G. Genette (1982: 8).

14. M. Riffaterre $(1980: 5$ et $13 ; 1983: 12)$.

15. L. Jenny (1976: 263).

16. M. Riffaterre (1983: 141).

17. C. Kerbrat-Orecchioni (1986: 161 et 194).

18. L. Jenny (1976: 266).

19. Lettre du 8 juin 1976 à B. Kneubuhl, citée par J. Savigneau (1990), Marguerite Yourcenar, l'invention d'une vie, Gallimard, Paris, page 175.

20. U. Eco, article du Figaro, $1^{\text {er }}$ semestre 2001.

21. Et ce, même si l'on se contente, comme nous le faisons ici, d'envisager le cas de l'intertextualité «obligatoire», qui se distingue, selon Riffaterre, d'une intertextualité «aléatoire».

22. On pourra se référer à ce sujet à l'étude de C. Nord (1993).

23. Par exemple dans Ezéchiel.

24. ATLAS, Septièmes assises de la traduction littéraire, 1990, Actes Sud, page 32.

25. Traduction Louis Segond.

26. Cité par G. Mounin (1994: 75).

27. Traduction de Marthe Robert.

28. W. E. Emrich (1970): Franz Kafka, Athenäum Verlag, Frankfurt/Bonn.

29. La réalité hypertextuelle de l'œuvre de Kafka a été, sans doute volontairement, occultée par Max Brod, auquel Vialatte puis les «exégètes» des années 1950 ont fait écho en France. Le fait que Kafka n'ait pas été, intertextuellement parlant, un écrivain «venu de nulle part» a été connu du public français à la suite des études universitaires menées dans les années 1960. C'est à partir de ce moment que les retraductions auraient dû le prendre en compte.

30. Même si la Note de l'éditeur tente d'amortir ce choc, en prévenant que les traducteurs «ne se sont pas interdit de s'éloigner en quelques occasions des sentiers battus en empruntant au français contemporain des expressions ou des tournures plus vives qu'il n'est généralement admis ».

31. Ce phénomène de rétroaction mériterait une étude pour lui seul. Car s'il est clair qu'une traduction peut opérer la modification rétroactive que nous signalons, il reste à savoir, inversement, dans quelles conditions sa lecture peut être modifiée par l'évolution ultérieure du champ intertextuel: pour A. Topia, «alors que [l'original] ne cesse de se ré-ajuster à l'intérieur d'une configuration toujours en mouvement, la traduction ne «bouge» pas», elle est «figée dans un temps verrouillé une fois pour toutes» (1990: 46). Sur ce sujet, on consultera également les remarques d'A. Brisset (2003).

32. Ici, nous rejoignons A. Topia qui signale, reprenant l'expression de Berman: «Les "grandes traductions », celles qui non seulement font date, mais réagissent sur les œuvres au milieu desquelles elles apparaissent, sont celles qui ont un intertexte, ou qui, par un effet de feedback, s'en constituent un » (1990: 48).

\section{RÉFÉRENCES}

Barthes, R. (1973-1995): "Théorie du texte», Encyclopédie Universalis.

Berman, A. (1999): L'épreuve de l'étranger, Gallimard, Paris.

Brisset, A. (2003): "Retraduire ou le corps changeant de la connaissance», Palimpsestes 15, Paris.

Folkart, B. (1991): Le conflit des énonciations, Traduction et discours rapporté, Les Éditions Balzac, Québec.

Genette, G. (1982): Palimpsestes, la littérature au second degré, Seuil, Paris.

Gouanvic, J.-M. (2001): «Ethos, éthique et traduction: vers une communauté de destin dans les cultures », TTR 14-2.

Iser, W (1985): L'acte de lecture, Mardaga, Bruxelles. 
IsRAËL, F. (2002): «La trace du lien en traduction», Identité, altérité, équivalence? La traduction comme relation, Lettres modernes Minard, Paris-Caen.

Jenny, L. (1976): «La stratégie de la forme», Poétique 27, Paris.

Kerbrat-Orecchioni, C. (1986): L'implicite, A. Colin, Paris.

Kristeva, J. (1969): «Le mot, le dialogue et le roman », Sèméiotikè, Seuil.

Maingueneau, D. (1976): Initiation aux Méthodes de l'analyse du discours, Hachette, Paris.

Mounin, G. (1994): Les belles infidèles, Presses Universitaires de Lille.

Nord, C. (1993): Einführung in das funktionale Übersetzen, Francke, Tübingen-Basel.

Riffaterre, M. (1971): Essais de stylistique structurale, Flammarion, Paris.

Riffaterre, M. (1979): La production du texte, Seuil, Paris.

Riffaterre, M. (1980): «La trace de l'intertexte», La Pensée, Paris.

Riffaterre, M.(1983): Sémiotique de la poésie, Seuil, Paris.

Robert, M. (1973): «Kafka en France», Obliques 3, Paris.

Sollers, Ph. (1968): Théorie d'ensemble, Seuil, Paris.

Topia, A. (1990): «Finnegans Wake: la traduction parasitée», Palimpsestes 4, Paris.

\section{Exemples}

Dickens, Ch. (1981): David Copperfield, Oxford University Press, Oxford.

Fontane, Th. (1969): Effi Briest, éd. Reclam, Stuttgart.

Fontane, Th. (1981): Effi Briest, tr. A. Couroy, Gallimard, Paris.

Fontane, Th. (1981): Effi Briest, tr. P. Villain, Romans, éd. M.F. Demet, pr. C. David, Robert Laffont, Paris.

Fontane, Th. (19xx) : Effi Briest, tr. H. Morrison and H. Chambers, Penguin Books, London.

Grass, G. (1995): Ein weites Feld, dtv, München.

Grass, G. (1997): Toute une histoire, tr. C. Porcell et B. Lortholary, Seuil, Paris.

Grass, G. (2000): Too Far Afield, tr. K. Winston, Faber and Faber, London.

KAFKa, F. (1994): Der Verschollene, Fischer, Ffurt/M.

KAFKA, F. (1976): «Le chauffeur», tr. Vialatte, Euvres complètes, Gallimard, Paris.

KAFKA, F. (1997): La métamorphose, La sentence, Le soutier et autres récits, tr. C. Billmann et J. Cellard, Actes Sud, Arles.

KAFKA, F. (2000): «Le soutier», tr. F. Mathieu, Récits, romans, journaux, La Pochothèque, Paris.

Mishima, Y. (1961): Le Pavillon d'or, tr. M. Mécréant, Gallimard, Paris.

Zweig, S. (1956): «Der Amokläufer», Amok, Novellen einer Leidenschaft, Fischer Verlag, Ffurt/ Main.

Zweig, S. (1927): Amok ou le fou de Malaisie, tr. Alzir Hella et O. Bournac, Stock, Paris.

Zweig, S. (1979): Amok ou le fou de Malaisie, tr. Alzir Hella et O. Bournac, revue par B. VergneCain et G. Rudent, Le Livre de poche, Paris. 